\title{
Multivariate sampling Kantorovich operators: quantitative estimates in Orlicz spaces
}

\author{
Laura Angeloni, Nursel Çetin, Danilo Costarelli, Anna Rita Sambucini, \\ AND GIANLUCA VINTI*
}

\begin{abstract}
In this paper, we establish a quantitative estimate for multivariate sampling Kantorovich operators by means of the modulus of smoothness in the general setting of Orlicz spaces. As a consequence, the qualitative order of convergence can be obtained, in case of functions belonging to suitable Lipschitz classes. In the particular instance of $L^{p}$-spaces, using a direct approach, we obtain a sharper estimate than that one that can be deduced from the general case.
\end{abstract}

Keywords: Multivariate sampling Kantorovich operators, Orlicz spaces, modulus of smoothness, quantitative estimates, Lipschitz classes.

2020 Mathematics Subject Classification: 41A25, 41A35.

Dedicated to Professor Francesco Altomare, on occasion of his 70th birthday, with esteem and friendship.

\section{INTRODUCTION}

The theory of generalized sampling-type operators is known since the 80 's, when it has been introduced by P. L. Butzer and his school with the aim to study approximate version of the celebrated Whittaker-Kotel'nikov-Shannon sampling theorem. It is closely related to positive linear operators and therefore this work fits into a field of Approximation Theory in which Prof. Francesco Altomare, to whom this paper is dedicated, has given basic and fundamental results (see, e.g., [2-5]).

Considering the long relationship of esteem, sharing of scientific interests and friendship by many of the authors of this paper with Prof. Francesco Altomare, it was an honor for us to have been invited to write this contribution dedicated to him. He was and will certainly continue to be a leading exponent of the Approximation Theory in Italy and abroad.

The Kantorovich version of the sampling-type operators has been introduced in [13], in onedimensional setting, with the aim to provide a family of linear operators suitable in order to reconstruct not necessarily continuous signals. Indeed, approximation results have been established in the very general context of Orlicz spaces ( $[12,32-34])$, in which are included the $L^{p}$-spaces and several other cases of well-known function spaces. Later on, a very complete theoretical study on all the above operators have been given, see [1, 6-8, 10, 15, 22, 29, 30, 35]. For instance, saturation results and inverse theorems of approximation have been established in $[14,26-28]$.

Received: 25.01.2021; Accepted: 16.02.2021; Published Online: 01.03.2021

*Corresponding author: Gianluca Vinti; gianluca.vinti@unipg.it

DOI: $10.33205 / \mathrm{cma} .876890$ 
The multivariate version of the above operators has been treated in [24] (see also [11]). This revealed to be very useful in order to face the problem of image reconstruction in several applied fields, since digital images are typical examples of multivariate discontinuous signals. For more details and, as concerns some applications of the above theory to concrete problems, the readers can see $[9,16,20,21]$.

In the present paper, a quantitative estimate for the multivariate sampling Kantorovich operators has been proved for functions belonging to Orlicz spaces. In order to get the above task, a suitable definition of the modulus of smoothness, based on the modular of the space, has been employed. We recall that the concept of modular arises from the theory of the modular spaces (see, e.g., [12,32]), which represent a further generalization of the Orlicz spaces.

Moreover, let us remark that the results proved in the present paper contain, as particular cases, some of the results established in [25] in the one-dimensional setting.

Formulating the prefixed results in the setting of Orlicz spaces allows a unifying approach that naturally includes results in $L^{p}$-spaces, Zygmund spaces, exponential spaces, and others; see, e.g., [12,25,32-34].

In particular, if we face the above problem in the $L^{p}$-case (that coincides with the Orlicz space generated by the $\varphi$-function $\varphi(u)=u^{p}$ ) by a direct approach, the modulus of smoothness of the Orlicz space reduces to the usual $L^{p}$-modulus of smoothness $\omega(f, \delta)_{p}$. This allows to exploit the well-known properties of $\omega(f, \delta)_{p}$ in order to establish an estimate that turns out to be sharper than that one achieved in the general (Orlicz) case.

Finally, the qualitative versions of the above results have been obtained assuming the involved functions in suitable Lipschitz classes.

\section{PRELIMINARY Notions}

We begin this section recalling the general setting of Orlicz spaces, in which we will work. First, we recall the notion of $\varphi$-function. A function $\varphi: \mathbb{R}_{0}^{+} \rightarrow \mathbb{R}_{0}^{+}$is said to be a $\varphi$-function if it satisfies the following conditions:

$\left(\Phi_{1}\right) \varphi$ is continuous and non-decreasing on $\mathbb{R}_{0}^{+}$;

$\left(\Phi_{2}\right) \varphi(0)=0, \varphi(u)>0$ for every $u>0$;

$\left(\Phi_{3}\right) \lim _{u \rightarrow+\infty} \varphi(u)=+\infty$.

For any fixed $\varphi$-function $\varphi$, we introduce the following modular functional $I^{\varphi}: M\left(\mathbb{R}^{n}\right) \rightarrow$ $[0,+\infty]$ defined by

$$
I^{\varphi}[f]:=\int_{\mathbb{R}^{n}} \varphi(|f(\underline{x})|) d \underline{x}
$$

for every $f \in M\left(\mathbb{R}^{n}\right)$, where here $M\left(\mathbb{R}^{n}\right)$ denotes the set of all Lebesgue measurable functions $f: \mathbb{R}^{n} \rightarrow \mathbb{R}$. Then, the Orlicz space generated by a $\varphi$ function $\varphi$ is defined by

$$
L^{\varphi}\left(\mathbb{R}^{n}\right):=\left\{f \in M\left(\mathbb{R}^{n}\right): I^{\varphi}[\lambda f]<+\infty \text { for some } \lambda>0\right\} .
$$

Now, we can recall a well-known concept of convergence in Orlicz spaces, i.e., the modular convergence (see, e.g., $[12,31,32])$. We say that a net of functions $\left(f_{w}\right)_{w>0} \subset L^{\varphi}\left(\mathbb{R}^{n}\right)$ is modularly convergent to a function $f \in L^{\varphi}\left(\mathbb{R}^{n}\right)$, if

$$
\lim _{w \rightarrow+\infty} I^{\varphi}\left[\lambda\left(f_{w}-f\right)\right]=\lim _{w \rightarrow+\infty} \int_{\mathbb{R}^{n}} \varphi\left(\lambda\left|f_{w}(\underline{x})-f(\underline{x})\right|\right) d \underline{x}=0
$$

for some $\lambda>0$. Now, in order to establish a quantitative estimate for the order of approximation of a family of linear multivariate operators, we recall the definition of the modulus 
of smoothness in Orlicz spaces $L^{\varphi}\left(\mathbb{R}^{n}\right)$. For any fixed $f \in M\left(\mathbb{R}^{n}\right)$ and $\delta>0$, we define the Orlicz-type modulus of smoothness by

$$
\omega(f, \delta)_{\varphi}:=\sup _{\|\underline{t}\|_{2} \leq \delta} I^{\varphi}[f(\cdot+\underline{t})-f(\cdot)]
$$

where $\|\cdot\|_{2}$ denotes the usual Euclidean norm of $\mathbb{R}^{n}$. It is well-known (see [12, Theorem 2.4] ) that if $f \in L^{\varphi}\left(\mathbb{R}^{n}\right)$, then there exists $\lambda>0$ such that

$$
\lim _{\delta \rightarrow 0} \omega(\lambda f, \delta)_{\varphi}=0 .
$$

Now, we recall the definition of the class of operators we work with. Let $\Pi^{n}=\left(t_{\underline{k}}\right)_{\underline{k} \in \mathbb{Z}^{n}} \subset \mathbb{R}^{n}$ be a sequence defined by $t_{\underline{k}}=\left(t_{k_{1}}, \ldots, t_{k_{n}}\right)$, where each $\left(t_{k_{i}}\right)_{k_{i} \in \mathbb{Z}}, i=1, \ldots, n$ is a sequence of real numbers such that $-\infty<t_{k_{i}}<t_{k_{i}+1}<+\infty, \lim _{k_{i} \rightarrow \pm \infty} t_{k_{i}}= \pm \infty$ for every $i=1, \ldots, n$ and there are two positive constants $\Delta, \delta$ for which $\delta \leq \Delta_{k_{i}}:=t_{k_{i}+1}-t_{k_{i}} \leq \Delta$ for every $i=1, \ldots, n$. Moreover, we denote by

$$
R_{w, t_{\underline{k}}}:=\left[\frac{t_{k_{1}}}{w}, \frac{t_{k_{1}+1}}{w}\right] \times \ldots \times\left[\frac{t_{k_{n}}}{w}, \frac{t_{k_{n}+1}}{w}\right], \quad(w>0),
$$

the $n$-dimensional interval associated to the sequence $\Pi^{n}$. For the sake of simplicity, we denote the Lebesgue measure of any $R_{w, t_{k}}$ by $A_{\underline{k}} / w^{n}$, where $A_{\underline{k}}:=\Delta_{k_{1}} \cdot \Delta_{k_{2}} \cdot \ldots \cdot \Delta_{k_{n}}$. In general, sequences of the form $\Pi^{n}$ are not necessarily equally distributed on $\mathbb{R}^{n}$, in fact producing a non-uniform sampling scheme. Clearly, if we consider the sequence $t_{\underline{k}}=\underline{k}, \underline{k} \in \mathbb{Z}^{n}$, we obtain an equally spaced grid of nodes, with $\delta=\Delta=\Delta_{k_{i}}=1$ and $A_{\underline{k}}=1$ for every $\underline{k} \in \mathbb{Z}^{n}$. From now on, a function $\chi: \mathbb{R}^{n} \rightarrow \mathbb{R}$ will be called a kernel if it satisfies the following conditions:

$\left(\chi_{1}\right) \quad \chi \in L^{1}\left(\mathbb{R}^{n}\right)$ and is bounded in a neighborhood of $\underline{0} \in \mathbb{R}^{n}$;

$\left(\chi_{2}\right)$ for every $\underline{u} \in \mathbb{R}^{n}$, with $\underline{u}=\left(u_{1}, \ldots, u_{n}\right)$, we have

$$
\sum_{\underline{k} \in \mathbb{Z}^{n}} \chi\left(\underline{u}-t_{\underline{k}}\right)=1
$$

$\left(\chi_{3}\right)$ for some $\beta>0$,

$$
m_{\beta, \Pi^{n}}(\chi):=\sup _{\underline{u} \in \mathbb{R}^{n}} \sum_{\underline{k} \in \mathbb{Z}^{n}}\left|\chi\left(\underline{u}-t_{\underline{k}}\right)\right|\left\|\underline{u}-t_{\underline{k}}\right\|_{2}^{\beta}<+\infty,
$$

i.e., the discrete absolute moment of order $\beta$ of $\chi$ is finite.

For examples of multivariate kernels see, e.g., [24]. We recall that, several (but not all) examples of multivariate kernels are defined as the product of $n$ one-dimensional kernels, such as the Fejér kernel, the central B-splines, and many others ( [17-19]). Now, we recall the following lemma that will be useful in the proof of the results of the next section.

Lemma 2.1. (see [24]) Let $\chi$ be a kernel satisfying conditions $\left(\chi_{1}\right)$ and $\left(\chi_{3}\right)$. Then, we have

$$
m_{0, \Pi^{n}}(\chi):=\sup _{\underline{u} \in \mathbb{R}^{n}} \sum_{\underline{k} \in \mathbb{Z}^{n}}\left|\chi\left(\underline{u}-t_{\underline{k}}\right)\right|<+\infty,
$$

where the convergence of the series $\sum_{\underline{k} \in \mathbb{Z}^{n}}\left|\chi\left(\underline{u}-t_{\underline{k}}\right)\right|$ is uniform on the compact subsets of $\mathbb{R}^{n}$. 
Thus, for any given kernel $\chi$, the corresponding family of multivariate sampling Kantorovich operators is defined by

$$
\left(S_{w}^{\chi} f\right)(\underline{x}):=\sum_{\underline{k} \in \mathbb{Z}^{n}} \chi\left(w \underline{x}-t_{\underline{k}}\right)\left[\frac{w^{n}}{A_{\underline{k}}} \int_{R_{w, t_{\underline{k}}}} f(\underline{u}) d \underline{u}\right], \quad \underline{x} \in \mathbb{R}^{n},
$$

where $f: \mathbb{R}^{n} \rightarrow \mathbb{R}$ is a locally integrable function such that the above series is convergent for every $\underline{x} \in \mathbb{R}^{n}$. It is well-known that the above operators are well-defined, for instance, if $f \in L^{\infty}(\mathbb{R})$, or if $f \in L^{\varphi}(\mathbb{R})$, where $\varphi$ is any convex $\varphi$-function (see [24]). In particular, in the setting of Orlicz spaces, the modular convergence of the family $S_{w}^{\chi} f$ to $f$ has been established in Theorem 4.5 of [24].

\section{MAin Results}

In this section, we establish a quantitative estimate for the multivariate sampling Kantorovich operators using the modulus of smoothness in Orlicz spaces $L^{\varphi}\left(\mathbb{R}^{n}\right)$, recalled in Section 2.

Theorem 3.1. Let $\varphi$ be a convex $\varphi$-function. Suppose that for any fixed $0<\alpha<1$, we have

$$
w^{n} \int_{\|\underline{y}\|_{2}>1 / w^{\alpha}}|\chi(w \underline{y})| d \underline{y} \leq M w^{-\gamma} \text {, as } w \rightarrow+\infty
$$

for suitable positive constants $M, \gamma$ depending on $\alpha$ and $\chi$. Then, for every $f \in L^{\varphi}\left(\mathbb{R}^{n}\right)$ and a suitable $\lambda_{f}=\lambda>0$, the following estimate holds:

$$
\begin{aligned}
I^{\varphi}\left[\lambda\left(S_{w}^{\chi} f-f\right)\right] & \leq \frac{\|\chi\|_{1}}{2 \delta^{n} m_{0, \Pi^{n}}(\chi)} \omega\left(2 m_{0, \Pi^{n}}(\chi) f, \frac{1}{w^{\alpha}}\right)_{\varphi} \\
& +\frac{M I^{\varphi}\left[4 \lambda m_{0, \Pi^{n}}(\chi) f\right]}{2 \delta^{n} m_{0, \Pi^{n}}(\chi)} w^{-\gamma} \\
& +\frac{\Delta^{n}}{2 \delta^{n}} \omega\left(2 m_{0, \Pi^{n}}(\chi) f, \sqrt{n} \frac{\Delta}{w}\right)_{\varphi}
\end{aligned}
$$

for every sufficiently large $w>0$, where $m_{0, \Pi^{n}}(\chi)<+\infty$ in view of Lemma 2.1. In particular, if $\lambda>0$ is sufficiently small, this inequality implies the modular convergence of multivariate sampling Kantorovich operators $S_{w}^{\chi} f$ to $f$.

Proof. Let $\lambda>0$ be fixed. Taking into account that $\varphi$ is convex and non-decreasing, we have

$$
\begin{aligned}
& I^{\varphi}\left[\lambda\left(S_{w}^{\chi} f-f\right)\right] \\
\leq & \frac{1}{2}\left\{\int_{\mathbb{R}^{n}} \varphi\left(2 \lambda\left|\left(S_{w}^{\chi} f\right)(\underline{x})-\sum_{\underline{k} \in \mathbb{Z}^{n}} \chi\left(w \underline{x}-t_{\underline{k}}\right) \frac{w^{n}}{A_{\underline{k}}} \int_{R_{w, t_{\underline{k}}}} f\left(\underline{u}+\underline{x}-\frac{t_{\underline{k}}}{w}\right) d \underline{u}\right|\right) d \underline{x}\right. \\
+ & \left.\int_{\mathbb{R}^{n}} \varphi\left(2 \lambda\left|\sum_{\underline{k} \in \mathbb{Z}^{n}} \chi\left(w \underline{x}-t_{\underline{k}}\right) \frac{w^{n}}{A_{\underline{k}}} \int f\left(\underline{u}+\underline{x}-\frac{t_{\underline{k}}}{w}\right) d \underline{u}-f(\underline{x})\right|\right) d \underline{x}\right\} \\
= & : J_{1}+J_{2} .
\end{aligned}
$$


Now, we estimate $J_{1}$. Using Jensen inequality twice (see, e.g., [23]), the change of variable $\underline{y}=\underline{x}-\frac{t_{\underline{k}}}{w}$, and Fubini-Tonelli theorem, we obtain

$$
\begin{aligned}
2 J_{1} & \leq \int_{\mathbb{R}^{n}} \varphi\left(2 \lambda \sum_{\underline{k} \in \mathbb{Z}^{n}}\left|\chi\left(w \underline{x}-t_{\underline{k}}\right)\right| \frac{w^{n}}{A_{\underline{k}}} \int_{R_{w, t_{\underline{k}}}}\left|f(\underline{u})-f\left(\underline{u}+\underline{x}-\frac{t_{\underline{k}}}{w}\right)\right| d \underline{u}\right) d \underline{x} \\
& \leq \frac{1}{m_{0, \Pi^{n}}(\chi)} \int_{\mathbb{R}^{n}} \sum_{\underline{k} \in \mathbb{Z}^{n}}\left|\chi\left(w \underline{x}-t_{\underline{k}}\right)\right| \varphi\left(2 \lambda m_{0, \Pi^{n}}(\chi) \frac{w^{n}}{A_{\underline{k}}} \int\left|f(\underline{u})-f\left(\underline{u}+\underline{x}-\frac{t_{\underline{k}}}{w}\right)\right| d \underline{u}\right) d \underline{x} \\
& \leq \frac{1}{m_{0, \Pi^{n}}(\chi)} \int_{\mathbb{R}^{n}} \sum_{\underline{k} \in \mathbb{Z}^{n}}\left|\chi\left(w \underline{x}-t_{\underline{k}}\right)\right| \frac{w^{n}}{A_{\underline{k}}} \int_{R_{w, t_{\underline{k}}}} \varphi\left(2 \lambda m_{0, \Pi^{n}}(\chi)\left|f(\underline{u})-f\left(\underline{u}+\underline{x}-\frac{t_{\underline{k}}}{w}\right)\right|\right) d \underline{u} d \underline{x} \\
& \leq \frac{1}{m_{0, \Pi^{n}}(\chi) \delta^{n}} \int_{\mathbb{R}^{n}}|\chi(w \underline{y})| w^{n} \sum_{\underline{k} \in \mathbb{Z}^{n} R_{w, t_{\underline{k}}}} \int_{\mathbb{R}\left(2 \lambda m_{0, \Pi^{n}}(\chi)|f(\underline{u})-f(\underline{u}+\underline{y})|\right) d \underline{u} d \underline{y}} \\
& =\frac{1}{m_{0, \Pi^{n}}(\chi) \delta^{n}} \int_{\mathbb{R}^{n}}|\chi(w \underline{y})| w^{n} \int_{\mathbb{R}^{n}} \varphi\left(2 \lambda m_{0, \Pi^{n}}(\chi)|f(\underline{u})-f(\underline{u}+\underline{y})|\right) d \underline{u} d \underline{y} \\
& =\frac{1}{m_{0, \Pi^{n}}(\chi) \delta^{n}} \int_{\mathbb{R}^{n}} w^{n}|\chi(w \underline{y})| I^{\varphi}\left[2 \lambda m_{0, \Pi^{n}}(\chi)(f(\cdot)-f(\cdot+\underline{y}))\right] d \underline{y} .
\end{aligned}
$$

Now, let $0<\alpha<1$ be fixed. We now split the above integral as follows:

$$
\begin{aligned}
& \frac{1}{m_{0, \Pi^{n}}(\chi) \delta^{n}}\left\{\int_{\|\underline{y}\|_{2} \leq 1 / w^{\alpha}}+\int_{\|\underline{y}\|_{2}>1 / w^{\alpha}}\right\} w^{n}|\chi(w \underline{y})| I^{\varphi}\left[2 \lambda m_{0, \Pi^{n}}(\chi)(f(\cdot)-f(\cdot+\underline{y}))\right] d \underline{y} \\
= & : J_{1,1}+J_{1,2} .
\end{aligned}
$$

For $J_{1,1}$, one has

$$
\begin{aligned}
J_{1,1} & \leq \frac{1}{m_{0, \Pi^{n}}(\chi) \delta^{n}} \int_{\|\underline{y}\|_{2} \leq 1 / w^{\alpha}} w^{n}|\chi(w \underline{y})| \omega\left(2 m_{0, \Pi^{n}}(\chi) f,\|\underline{y}\|_{2}\right)_{\varphi} d \underline{y} \\
& \leq \omega\left(2 m_{0, \Pi^{n}}(\chi) f, \frac{1}{w^{\alpha}}\right)_{\varphi} \frac{w^{n}}{m_{0, \Pi^{n}}(\chi) \delta^{n}} \int_{\|\underline{y}\|_{2} \leq 1 / w^{\alpha}}|\chi(w \underline{y})| d \underline{y} \\
& \leq \omega\left(2 m_{0, \Pi^{n}}(\chi) f, \frac{1}{w^{\alpha}}\right)_{\varphi} \frac{\|\chi\|_{1}}{m_{0, \Pi^{n}}(\chi) \delta^{n}} .
\end{aligned}
$$

On the other hand, taking into account that $\varphi$ is convex, for $J_{1,2}$, we can write

$$
\begin{aligned}
J_{1,2} & \leq \frac{1}{m_{0, \Pi^{n}}(\chi) \delta^{n}} \int_{\|\underline{y}\|_{2}>1 / w^{\alpha}} w^{n}|\chi(w \underline{y})| \frac{1}{2}\left\{I^{\varphi}\left[4 \lambda m_{0, \Pi^{n}}(\chi) f(\cdot)\right]\right. \\
& \left.+I^{\varphi}\left[4 \lambda m_{0, \Pi^{n}}(\chi) f(\cdot+\underline{y})\right]\right\} d \underline{y} .
\end{aligned}
$$


Moreover, it can be easily seen that

$$
I^{\varphi}\left[4 \lambda m_{0, \Pi^{n}}(\chi) f(\cdot)\right]=I^{\varphi}\left[4 \lambda m_{0, \Pi^{n}}(\chi) f(\cdot+\underline{y})\right]
$$

for every $\underline{y}$. Therefore, by assumption (3.3), we finally have

$$
\begin{aligned}
J_{1,2} & \leq \frac{1}{m_{0, \Pi^{n}}(\chi) \delta^{n}} \int_{\|\underline{y}\|_{2}>1 / w^{\alpha}} w^{n}|\chi(w \underline{y})| I^{\varphi}\left[4 \lambda m_{0, \Pi^{n}}(\chi) f(\cdot)\right] d \underline{y} \\
& \leq \frac{I^{\varphi}\left[4 \lambda m_{0, \Pi^{n}}(\chi) f\right]}{m_{0, \Pi^{n}}(\chi) \delta^{n}} M w^{-\gamma}
\end{aligned}
$$

for $w>0$ sufficiently large. Now, we can estimate $J_{2}$. Using the singularity assumption $\left(\chi_{2}\right)$, we immediately have

$$
2 J_{2} \leq \int_{\mathbb{R}^{n}} \varphi\left(2 \lambda \sum_{\underline{k} \in \mathbb{Z}^{n}}\left|\chi\left(w \underline{x}-t_{\underline{k}}\right)\right| \frac{w^{n}}{A_{\underline{k}}} \int_{R_{w, t_{\underline{k}}}}\left|f\left(\underline{u}+\underline{x}-\frac{t_{\underline{k}}}{w}\right)-f(\underline{x})\right| d \underline{u}\right) d \underline{x} .
$$

Now, using twice Jensen inequality as above and the change of variable $\underline{y}=\underline{u}-\frac{t_{\underline{k}}}{w}$, we obtain

$$
\begin{aligned}
2 J_{2} & \leq \frac{1}{m_{0, \Pi^{n}}(\chi)} \int_{\mathbb{R}^{n}} \sum_{\underline{k} \in \mathbb{Z}^{n}}\left|\chi\left(w \underline{x}-t_{\underline{k}}\right)\right| \frac{w^{n}}{A_{\underline{k}}} \int_{R_{w, t_{\underline{k}}}} \varphi\left(2 \lambda m_{0, \Pi^{n}}(\chi)\left|f\left(\underline{u}+\underline{x}-\frac{t_{\underline{k}}}{w}\right)-f(\underline{x})\right|\right) d \underline{u} d \underline{x} \\
& \leq \frac{1}{m_{0, \Pi^{n}}(\chi) \delta^{n}} \int_{\mathbb{R}^{n}} \sum_{\underline{k} \in \mathbb{Z}^{n}}\left|\chi\left(w \underline{x}-t_{\underline{k}}\right)\right| w^{n} \int \varphi\left(2 \lambda m_{0, \Pi^{n}}(\chi)|f(\underline{x}+\underline{y})-f(\underline{x})|\right) d \underline{y} d \underline{x}, \\
\left(R_{w, t_{\underline{k}}}-\frac{t_{\underline{k}}}{w}\right) &
\end{aligned}
$$

where the symbol $\left(R_{w, t_{\underline{k}}}-\frac{t_{\underline{k}}}{w}\right):=\left[0, \frac{\Delta_{k_{1}}}{w}\right] \times \ldots \times\left[0, \frac{\Delta_{k_{n}}}{w}\right]$ for every $\underline{k} \in \mathbb{Z}^{n}$ and $w>0$.

Hence, by the Fubini-Tonelli theorem,

$$
\begin{aligned}
2 J_{2} & \leq \frac{1}{\delta^{n}} \int_{\mathbb{R}^{n}} w^{n} \int \frac{t^{\prime}}{\left(R_{w, t_{\underline{k}}}-\frac{t_{\underline{k}}}{w}\right)} \varphi\left(2 \lambda m_{0, \Pi^{n}}(\chi)|f(\underline{x}+\underline{y})-f(\underline{x})|\right) d \underline{y} d \underline{x} \\
& \leq \frac{w^{n}}{\delta^{n}} \int I^{\varphi}\left[2 \lambda m_{0, \Pi^{n}}(\chi)(f(\cdot+\underline{y})-f(\cdot))\right] d \underline{y} \\
& \leq \frac{w^{n}}{\delta^{n}} \int_{\left(\Delta_{w}\right)} I^{\varphi}\left[2 \lambda m_{0, \Pi^{n}}(\chi)(f(\cdot+\underline{y})-f(\cdot))\right] d \underline{y},
\end{aligned}
$$


where $\left(\Delta_{w}\right):=\left[0, \frac{\Delta}{w}\right] \times \ldots \times\left[0, \frac{\Delta}{w}\right]$. Then, we get

$$
\begin{aligned}
2 J_{2} & \leq \frac{w^{n}}{\delta^{n}} \int_{\left(\Delta_{w}\right)} \omega\left(2 m_{0, \Pi^{n}}(\chi) f, \sqrt{n} \frac{\Delta}{w}\right)_{\varphi} d \underline{y} \\
& \leq \frac{\Delta^{n}}{\delta^{n}} \omega\left(2 m_{0, \Pi^{n}}(\chi) f, \sqrt{n} \frac{\Delta}{w}\right)_{\varphi},
\end{aligned}
$$

which completes the proof.

\section{Remark 3.1.}

(1) Note that, it is easy to show that for any kernels such that $\chi(\underline{u})=\mathcal{O}\left(\|\underline{u}\|_{2}^{-\theta}\right)$, as $\|\underline{u}\|_{2} \rightarrow$ $+\infty$, for $\theta>1$, we have that assumption (3.3) is satisfied for some constant $M>0$ and $\gamma=(1-\alpha)(\theta-1)>0$ for every fixed $0<\alpha<1$ (see, e.g., [25]).

(2) For further examples of kernels satisfying assumption (3.3), with and without compact support, the reader can refer to [15].

Now, we recall the definition of Lipschitz classes in Orlicz spaces $L^{\varphi}\left(\mathbb{R}^{n}\right)$. We define by $\operatorname{Lip}_{\varphi}(\nu), 0<\nu \leq 1$, the set of all functions $f \in M\left(\mathbb{R}^{n}\right)$ such that there exists $\lambda>0$ with

$$
I^{\varphi}[\lambda(f(\cdot)-f(\cdot+\underline{t}))]=\int_{\mathbb{R}^{n}} \varphi(\lambda|f(\underline{x})-f(\underline{x}+\underline{t})|) d \underline{x}=O\left(\|\underline{t}\|_{2}^{\nu}\right)
$$

as $\|\underline{t}\|_{2} \rightarrow 0$. From Theorem 3.1, we immediately obtain the following corollary.

Corollary 3.1. Under the assumptions of Theorem 3.1 with $0<\alpha<1$ and for any $f \in \operatorname{Lip}_{\varphi}(\nu)$, $0<\nu \leq 1$, there exist $K>0$ and $\lambda>0$ such that

$$
I^{\varphi}\left[\lambda\left(S_{w}^{\chi} f-f\right)\right] \leq K w^{-\theta}
$$

for sufficiently large $w>0$, where $\theta:=\min \{\alpha \nu, \gamma\}$.

Note that, the results established in Theorem 3.1 and Corollary 3.1 are valid in case of functions belonging to $L^{\varphi}\left(\mathbb{R}^{n}\right)$, with $\varphi$ convex. Hence, applications can be easily obtained in some well-known cases of Orlicz spaces, such as the $L^{p}$-spaces, the Zygmund (or interpolation) spaces, and the exponential spaces. For more details concerning the above instances of Orlicz spaces, see, e.g., [13,24].

Actually, in the particular case of $L^{p}$-spaces (i.e., when $\varphi(u)=u^{p}, u \in \mathbb{R}_{0}^{+}, p \geq 1$ ), thanks to the well-known properties of the first order modulus of smoothness in $L^{p}$, we can also establish the following direct quantitative estimate, which turns out to be sharper than that one established in the general case considered in Theorem 3.1 (and consequently also in Corollary 3.1).

In order to obtain the above mentioned result for the multivariate sampling Kantorovich operators, we recall, for $f \in L^{p}\left(\mathbb{R}^{n}\right)$, the definition of the $L^{p}$-first order modulus of smoothness of $f$, given by

$$
\omega(f, \delta)_{p}=\sup _{\|\underline{h}\|_{2} \leq \delta}\left(\int_{\mathbb{R}^{n}}|f(\underline{t}+\underline{h})-f(\underline{t})|^{p} d \underline{t}\right)^{1 / p},
$$

with $\delta>0,1 \leq p<+\infty$. We can prove the following estimate. 
Theorem 3.2. Suppose that

$$
M_{p}(\chi):=\int_{\mathbb{R}^{n}}|\chi(\underline{t})|\|\underline{t}\|_{2}^{p} d \underline{t}<+\infty
$$

for some $1 \leq p<+\infty$. Then, for every $f \in L^{p}\left(\mathbb{R}^{n}\right)$, the following quantitative estimate holds

$$
\begin{aligned}
\left\|S_{w}^{\chi} f-f\right\|_{p} & \leq \frac{\left[2 m_{0, \Pi^{n}}(\chi)\right]^{(p-1) / p}}{\delta^{n / p}}\left[\|\chi\|_{1}+M_{p}(\chi)\right]^{1 / p} \omega(f, 1 / w)_{p} \\
& +\left(\frac{\Delta}{\delta}\right)^{n / p} m_{0, \Pi^{n}}(\chi) \omega\left(f, \sqrt{n} \frac{\Delta}{w}\right)_{p}
\end{aligned}
$$

for every sufficiently large $w>0$.

Proof. Proceeding as in the first part of the proof of Theorem 3.1, and using the Minkowsky inequality, the concavity and hence the subadditivity of the function $|\cdot|^{1 / p}$, we have

$$
\begin{aligned}
\left\|S_{w}^{\chi} f-f\right\|_{p} & \leq\left(\int_{\mathbb{R}^{n}}\left[\sum_{\underline{k} \in \mathbb{Z}^{n}}\left|\chi\left(w \underline{x}-t_{\underline{k}}\right)\right| \frac{w^{n}}{A_{\underline{k}}} \int_{R_{w, t_{\underline{k}}}}\left|f(\underline{u})-f\left(\underline{u}+\underline{x}-\frac{t_{\underline{k}}}{w}\right)\right| d \underline{u}\right]^{p} d \underline{x}\right)^{1 / p} \\
& +\left(\int_{\mathbb{R}^{n}}\left[\sum_{\underline{k} \in \mathbb{Z}^{n}}\left|\chi\left(w \underline{x}-t_{\underline{k}}\right)\right| \frac{w^{n}}{A_{\underline{k}}} \int_{R_{w, t_{\underline{k}}}}\left|f\left(\underline{u}+\underline{x}-\frac{t_{\underline{k}}}{w}\right)-f(\underline{x})\right| d \underline{u}\right]^{p} d \underline{x}\right)^{1 / p} \\
& =: J_{1}+J_{2} .
\end{aligned}
$$

We now estimate $J_{1}$. Proceeding as in the proof of Theorem 3.1, i.e., applying Jensen inequality twice, Fubini-Tonelli theorem and the change of variable $\underline{y}=\underline{x}-\frac{t_{\underline{k}}}{w}$, we get

$$
\begin{aligned}
& J_{1}^{p}=\int_{\mathbb{R}^{n}}\left[\sum_{\underline{k} \in \mathbb{Z}^{n}}\left|\chi\left(w \underline{x}-t_{\underline{k}}\right)\right| \frac{w^{n}}{A_{\underline{k}}} \int_{R_{w, t_{\underline{k}}}}\left|f(\underline{u})-f\left(\underline{u}+\underline{x}-\frac{t_{\underline{k}}}{w}\right)\right| d \underline{u}\right]^{p} d \underline{x} \\
& \leq m_{0, \Pi^{n}}(\chi)^{p-1} \int_{\mathbb{R}^{n}} \sum_{\underline{k} \in \mathbb{Z}^{n}}\left|\chi\left(w \underline{x}-t_{\underline{k}}\right)\right|\left[\frac{w^{n}}{A_{\underline{k}}} \int_{R_{w, t_{\underline{k}}}}\left|f(\underline{u})-f\left(\underline{u}+\underline{x}-\frac{t_{\underline{k}}}{w}\right)\right| d \underline{u}\right]^{p} d \underline{x} \\
& \leq m_{0, \Pi^{n}}(\chi)^{p-1} \int_{\mathbb{R}^{n}} \sum_{\underline{k} \in \mathbb{Z}^{n}}\left|\chi\left(w \underline{x}-t_{\underline{k}}\right)\right|\left[\frac{w^{n}}{A_{\underline{k}}} \int_{R_{w, t_{\underline{k}}}}\left|f(\underline{u})-f\left(\underline{u}+\underline{x}-\frac{t_{\underline{k}}}{w}\right)\right|^{p} d \underline{u}\right] d \underline{x} \\
& \leq m_{0, \Pi^{n}}(\chi)^{p-1} \int_{\mathbb{R}^{n}} \sum_{\underline{k} \in \mathbb{Z}^{n}}|\chi(w \underline{y})|\left[\frac{w^{n}}{A_{\underline{k}}} \int_{R_{w, t_{\underline{k}}}}|f(\underline{u})-f(\underline{u}+\underline{y})|^{p} d \underline{u}\right] d \underline{y} \\
& \leq \frac{m_{0, \Pi^{n}}(\chi)^{p-1}}{\delta^{n}} \int_{\mathbb{R}^{n}} w^{n}|\chi(w \underline{y})|\left[\sum_{\underline{k} \in \mathbb{Z}^{n}} \int_{R_{w, t_{\underline{k}}}}|f(\underline{u})-f(\underline{u}+\underline{y})|^{p} d \underline{u}\right] d \underline{y}
\end{aligned}
$$




$$
\begin{aligned}
& =\frac{m_{0, \Pi^{n}}(\chi)^{p-1}}{\delta^{n}} \int_{\mathbb{R}^{n}} w^{n}|\chi(w \underline{y})|\left[\int_{\mathbb{R}^{n}}|f(\underline{u})-f(\underline{u}+\underline{y})|^{p} d \underline{u}\right] d \underline{y} \\
& \leq \frac{m_{0, \Pi^{n}}(\chi)^{p-1}}{\delta^{n}} \int_{\mathbb{R}^{n}} w^{n}|\chi(w \underline{y})| \omega\left(f,\|\underline{y}\|_{2}\right)_{p}^{p} d \underline{y} \\
& \leq \frac{m_{0, \Pi^{n}}(\chi)^{p-1}}{\delta^{n}} \int_{\mathbb{R}^{n}} w^{n}|\chi(w \underline{y})|\left(1+w\|\underline{y}\|_{2}\right)^{p} \omega\left(f, \frac{1}{w}\right)_{p}^{p} d \underline{y} \\
& \leq \frac{m_{0, \Pi^{n}}(\chi)^{p-1}}{\delta^{n}} 2^{p-1} \omega\left(f, \frac{1}{w}\right)_{p}^{p} \int_{\mathbb{R}^{n}} w^{n}|\chi(w \underline{y})|\left[1+\left(w\left\|_{y}\right\|_{2}\right)^{p}\right] d \underline{y} \\
& =\frac{m_{0, \Pi^{n}}(\chi)^{p-1}}{\delta^{n}} 2^{p-1} \omega\left(f, \frac{1}{w}\right)_{p}^{p}\left\{\int_{\mathbb{R}^{n}} w^{n}|\chi(w \underline{y})| d \underline{y}+\int_{\mathbb{R}^{n}} w^{n}|\chi(w \underline{y})|\left(w\left\|_{2}\right\|_{2}\right)^{p} d \underline{y}\right\} \\
& =\frac{m_{0, \Pi^{n}}(\chi)^{p-1}}{\delta^{n}} 2^{p-1} \omega\left(f, \frac{1}{w}\right)_{p}^{p}\left(\|\chi\|_{1}+M_{p}(\chi)\right)<+\infty
\end{aligned}
$$

for every $w>0$, where $\|\chi\|_{1}$ and $M_{p}(\chi)$ are both finite, in view of $\left(\chi_{1}\right)$ and (3.4). Note that, in the above estimates, we used the well-known inequality:

$$
\omega(f, \lambda \delta)_{p} \leq(1+\lambda) \omega(f, \delta)_{p}, \quad \lambda, \delta>0 .^{1}
$$

Now, we estimate $J_{2}$. Using Jensen inequality twice, the change of variable $\underline{y}=\underline{u}-\frac{t_{\underline{k}}}{w}$ and Fubini-Tonelli theorem, we have

$$
\begin{aligned}
& J_{2}^{p}=\int_{\mathbb{R}^{n}}\left[\sum_{\underline{k} \in \mathbb{Z}^{n}}\left|\chi\left(w \underline{x}-t_{\underline{k}}\right)\right| \frac{w^{n}}{A_{\underline{k}}} \int_{R_{w, t_{\underline{k}}}}\left|f\left(\underline{u}+\underline{x}-\frac{t_{\underline{k}}}{w}\right)-f(\underline{x})\right| d \underline{u}\right]^{p} d \underline{x} \\
& \leq \int_{\mathbb{R}^{n}}\left[\sum_{\underline{k} \in \mathbb{Z}^{n}}\left|\chi\left(w \underline{x}-t_{\underline{k}}\right)\right| \frac{w^{n}}{A_{\underline{k}}} \int_{\left(R_{w, t_{\underline{k}}}-\frac{t_{\underline{k}}}{w}\right)}|f(\underline{x}+\underline{y})-f(\underline{x})| d \underline{y}\right]^{p} d \underline{x}
\end{aligned}
$$



\footnotetext{
${ }^{1}$ In general, this inequality does not hold in the case of $\omega(f, \delta)_{\varphi}$ (i.e., in Orlicz spaces).
} 


$$
\begin{aligned}
& \leq \frac{m_{0, \Pi^{n}}(\chi)^{p-1}}{\delta^{n}} \int_{\mathbb{R}^{n}} \sum_{\underline{k} \in \mathbb{Z}^{n}}\left|\chi\left(w \underline{x}-t_{\underline{k}}\right)\right| w^{n}\left[\begin{array}{c}
\left.\int_{\left(R_{w, t_{\underline{k}}}-\frac{t_{\underline{k}}}{w}\right)}|f(\underline{x}+\underline{y})-f(\underline{x})|^{p} d \underline{y}\right] d \underline{x} \\
\leq \frac{m_{0, \Pi^{n}}(\chi)^{p}}{\delta^{n}} \int_{\mathbb{R}^{n}} w^{n}\left[\int_{\left(\Delta_{w}\right)}|f(\underline{x}+\underline{y})-f(\underline{x})|^{p} d \underline{y}\right] d \underline{x},
\end{array}\right]
\end{aligned}
$$

where $\left(\Delta_{w}\right):=\left[0, \frac{\Delta}{w}\right] \times \ldots \times\left[0, \frac{\Delta}{w}\right]$. Then, we obtain

$$
\begin{aligned}
J_{2}^{p} & \leq \frac{m_{0, \Pi^{n}}(\chi)^{p}}{\delta^{n}} \int_{\left(\Delta_{w}\right)} w^{n}\left[\int_{\mathbb{R}^{n}}|f(\underline{x}+\underline{y})-f(\underline{x})|^{p} d \underline{x}\right] d \underline{y} \\
& \leq \frac{m_{0, \Pi^{n}}(\chi)^{p}}{\delta^{n}} \int_{\left(\Delta_{w}\right)} w^{n}\left[\omega\left(f, \sqrt{n} \frac{\Delta}{w}\right)_{p}\right]^{p} d \underline{y} \\
& =\frac{m_{0, \Pi^{n}}(\chi)^{p}}{\delta^{n}} \Delta^{n}\left[\omega\left(f, \sqrt{n} \frac{\Delta}{w}\right)_{p}\right]^{p}
\end{aligned}
$$

This proves the theorem.

Note that also assumption (3.4) is quite standard and it is satisfied for kernels $\chi$ having sufficiently rapid decay, as for instance $\chi$ with compact support. Moreover, we remark that assumption (3.4) implies (3.3) but, in this context, (3.4) appears more natural to assume rather than (3.3). Also, note that what it allows us to achieve a sharper quantitative estimate depends only on the properties of the $L^{p}$-modulus of smoothness.

As made in the general context of Orlicz spaces, from the above quantitative estimate, we can directly deduce the qualitative order of approximation, assuming $f$ in suitable Lipschitz spaces.

Firstly, we recall that the Lipschitz class of Zygmund-type in $L^{p}$-spaces, with $0<\alpha \leq 1$, are defined as follows:

$$
\operatorname{Lip}(\alpha, p):=\left\{f \in L^{p}\left(\mathbb{R}^{n}\right):\|f(\cdot+\underline{t})-f(\cdot)\|_{p}=O\left(\|\underline{t}\|_{2}^{\alpha}\right), \text { as }\|\underline{t}\|_{2} \rightarrow 0\right\} \text {. }
$$

Now, we can state the following result.

Corollary 3.2. Suppose that

$$
M_{p}(\chi):=\int_{\mathbb{R}^{n}}|\chi(\underline{t})|\|\underline{t}\|_{2}^{p} d \underline{t}<+\infty
$$


for some $1 \leq p<+\infty$. Then, for every $f \in \operatorname{Lip}(\alpha, p), 0<\alpha \leq 1$, we have

$$
\begin{aligned}
\left\|S_{w}^{\chi} f-f\right\|_{p} & \leq \frac{\left[2 m_{0, \Pi^{n}}(\chi)\right]^{(p-1) / p}}{\delta^{n / p}}\left[\|\chi\|_{1}+M_{p}(\chi)\right]^{1 / p} C_{1} \frac{1}{w^{\alpha}} \\
& +\left(\frac{\Delta}{\delta}\right)^{n / p} m_{0, \Pi^{n}}(\chi) C_{1}\left(\sqrt{n} \frac{\Delta}{w}\right)^{\alpha}
\end{aligned}
$$

for every sufficiently large $w>0$, where $C_{1}>0$ is the constant coming from definition (3.5).

\section{ACKNOWLEDGMENTS}

The authors (except the second one) are members of the Gruppo Nazionale per l'Analisi Matematica, la Probabilità e le loro Applicazioni (GNAMPA) of the Istituto Nazionale di Alta Matematica (INdAM), of the network RITA (Research ITalian network on Approximation), and of the UMI group "Teoria dell' Approssimazione e Applicazioni".

The authors L. Angeloni, D. Costarelli, and A.R. Sambucini have been partially supported within the 2020 GNAMPA-INdAM Project "Analisi reale, teoria della misura ed approssimazione per la ricostruzione di immagini", while the authors L.Angeloni, A.R. Sambucini and G. Vinti within the projects: (1) Ricerca di Base 2017 dell'Università degli Studi di Perugia "Metodi di teoria degli operatori e di Analisi Reale per problemi di approssimazione ed applicazioni", (2) Ricerca di Base 2018 dell’Università degli Studi di Perugia - "Metodi di Teoria dell'Approssimazione, Analisi Reale, Analisi Nonlineare e loro Applicazioni", (3) "Metodi e processi innovativi per lo sviluppo di una banca di immagini mediche per fini diagnostici" funded by the Fondazione Cassa di Risparmio di Perugia, (FCRP), 2018, (4) "Metodiche di Imaging non invasivo mediante angiografia OCT sequenziale per lo studio delle Retinopatie degenerative dell' Anziano (M.I.R.A.)", funded by FCRP, 2019.

\section{REFERENCES}

[1] T. Acar, D. Costarelli and G. Vinti: Linear prediction and simultaneous approximation by m-th order Kantorovich type sampling series, Banach J. Math. Anal., 14 (4) (2020), 1481-1508.

[2] F. Altomare, M. Campiti: Korovkin-type approximation theory and its applications, De Gruyter studies in Mathematics, (2011).

[3] F. Altomare, M. Cappelletti Montano and V. Leonessa: On a Generalization of Szász-Mirakjan-Kantorovich Operators, Results Math., 63 (2013), 837-863.

[4] F. Altomare, M. Cappelletti Montano, V. Leonessa and I. Rasa: A generalization of Kantorovich operators for convex compact subsets, Banach J. Math. Anal., 11 (3) (2017), 591-614.

[5] F. Altomare, M. Cappelletti Montano, V. Leonessa and I. Rasa: Elliptic differential operators and positive semigroups associated with generalized Kantorovich operators, J. Math. Anal. Appl., 458 (1) (2018), 153-173.

[6] L. Angeloni, D. Costarelli, M. Seracini, G. Vinti and L. Zampogni: Variation diminishing-type properties for multivariate sampling Kantorovich operators, Bollettino U.M.I., Special issue dedicated to Prof. Domenico Candeloro, 13 (4) (2020), 595-605.

[7] L. Angeloni, D. Costarelli and G. Vinti: A characterization of the convergence in variation for the generalized sampling series, Ann. Acad. Sci. Fenn. Math., 43 (2018), 755-767.

[8] L. Angeloni, D. Costarelli and G. Vinti: Convergence in variation for the multidimensional generalized sampling series and applications to smoothing for digital image processing, Ann. Acad. Sci. Fenn. Math., 45 (2020), 751-770.

[9] F. Asdrubali, G. Baldinelli, F. Bianchi, D. Costarelli, A. Rotili, M. Seracini and G. Vinti: Detection of thermal bridges from thermographic images by means of image processing approximation algorithms, Appl. Math. Comput., 317 (2018), 160-171.

[10] C. Bardaro, I. Mantellini: Voronovskaya formulae for Kantorovich type generalized sampling series, Int. J. Pure Appl. Math., 62 (3) (2010), 247-262.

[11] C. Bardaro, I. Mantellini: Asymptotic formulae for multivariate Kantorovich type generalized sampling series, Acta Math. Sinica (ES), 27 (7) (2011), 1247-1258.

[12] C. Bardaro, J. Musielak and G. Vinti: Nonlinear Integral Operators and Applications, in: de Gruyter Series in Nonlinear Analysis and Applications, vol. 9, Walter de Gruyter \& Co., Berlin, (2003). 
[13] C. Bardaro, P. L. Butzer, R. L. Stens and G. Vinti: Kantorovich-type generalized sampling series in the setting of Orlicz spaces, Sampl. Theory Signal Image Process., 6 (1) (2007), 29-52.

[14] M. Cantarini, D. Costarelli and G. Vinti: A solution of the problem of inverse approximation for the sampling Kantorovich operators in case of Lipschitz functions, Dolomites Res. Notes Approx. DRNA, 13 (2020), 30-35.

[15] N. Çetin, D. Costarelli and G. Vinti: Quantitative estimates for nonlinear sampling Kantorovich operators, arXiv 2102.08651 (2021).

[16] F. Cluni, D. Costarelli, V. Gusella and G. Vinti: Reliability increase of masonry characteristics estimation by sampling algorithm applied to thermographic digital images, Probabilist Eng. Mech., 60 (2020), 103022.

[17] L. Coroianu, S. G. Gal: $L^{p}$-approximation by truncated max-product sampling operators of Kantorovich-type based on Fejer kernel, J. Integral Equations Applications, 29 (2) (2017), 349-364.

[18] L. Coroianu, S. G. Gal: Approximation by truncated max-product operators of Kantorovich-type based on generalized $(\Phi, \Psi)$-kernels, Math. Methods Appl. Sci., 41 (17) (2018), 7971-7984.

[19] L. Coroianu, S. G. Gal: Approximation by max-product operators of Kantorovich type, Stud. Univ. Babes-Bolyai Math., 64 (2) (2019), 207-223.

[20] D. Costarelli, M. Seracini and G. Vinti: A segmentation procedure of the pervious area of the aorta artery from CT images without contrast medium, Math. Methods Appl. Sci., 43 (2020), 114-133.

[21] D. Costarelli, M. Seracini and G. Vinti: A comparison between the sampling Kantorovich algorithm for digital image processing with some interpolation and quasi-interpolation methods, Appl. Math. Comput., 374 (2020), 125046.

[22] D. Costarelli, A. R. Sambucini and G. Vinti: Convergence in Orlicz spaces by means of the multivariate max-product neural network operators of the Kantorovich type, Neural Comput. \& Applic., 31 (9) (2019), 5069-5078.

[23] D. Costarelli, R. Spigler: How sharp is the Jensen inequality ?, J. Inequal. Appl., 2015:69 (2015) 1-10.

[24] D. Costarelli, G. Vinti: Approximation by Multivariate Generalized Sampling Kantorovich Operators in the Setting of Orlicz Spaces, Bollettino U.M.I., Special issue dedicated to Prof. Giovanni Prodi, 9 (4) (2011), 445-468.

[25] D. Costarelli, G. Vinti: A quantitative estimate for the sampling Kantorovich series in terms of the modulus of continuity in Orlicz spaces, Constr. Math. Anal., 2 (1) (2019), 8-14.

[26] D. Costarelli, G. Vinti: An inverse result of approximation by sampling Kantorovich series, Proc. Edinburgh Math. Soc., 62 (1) (2019), 265-280.

[27] D. Costarelli, G. Vinti: Inverse results of approximation and the saturation order for the sampling Kantorovich series, J. Approx. Theor., 242 (2019), 64-82.

[28] D. Costarelli, G. Vinti: Saturation by the Fourier transform method for the sampling Kantorovich series based on bandlimited kernels, Anal. Math. Phys., 9 (2019), 2263-2280.

[29] E. D'Aniello, M. Maiuriello: A survey on composition operators on some function spaces, Aequat. Math., (2020).

[30] A. Krivoshein, M. A. Skopina: Multivariate sampling-type approximation, Anal. Appl., 15 (4) (2017), 521-542.

[31] J. Musielak, W. Orlicz: On modular spaces, Studia Math., 28 (1959), 49-65.

[32] J. Musielak: Orlicz Spaces and Modular Spaces, in: Lecture Notes in Mathematics, vol. 1034, Springer-Verlag, Berlin, (1983).

[33] M. M. Rao, Z.D. Ren: Theory of Orlicz Spaces, Marcel Dekker Inc., Pure and Appl. Math., New York-Basel-Hong Kong, (1991).

[34] M. M. Rao, Z. D. Ren: Applications of Orlicz Spaces, Marcel Dekker Inc., Monographs and Textbooks in Pure and applied Mathematics, vol. 250, New York, (2002).

[35] G. Vinti, L. Zampogni: Approximation by means of nonlinear Kantorovich sampling type operators in Orlicz spaces, J. Approx. Theor., 161 (2009), 511-528.

\author{
LAURA ANGELONI \\ UNIVERSITY OF PERUGIA \\ DEPARTMENT OF MATHEMATICS AND COMPUTER SCIENCES \\ 1, Via VANVITELLi, 06123 PERUGia, ITALY \\ ORCID: 0000-0002-2214-6751 \\ E-mail address: laura . angeloni@unipg. it
}


NuRSEL ÇETIN

ANKARA HACI BAYRAM VELI UNIVERSITY

POlatli FACUlTy OF SCIENCE AND LETTERS

DEPARTMENT OF MATHEMATICS

06900, ANKARA, TURKEY

ORCID: 0000-0003-3771-6523

E-mail address: nurselcet in 07 @gmail.com

DANILO COSTARELLI

UNIVERSITY OF PERUGIA

DEPARTMENT OF MATHEMATICS AND COMPUTER SCIENCES

1, Via VANVITELli, 06123 Perugia, ItAly

ORCID: 0000-0001-8834-8877

E-mail address: danilo.costarelli@unipg.it

ANNA RITA SAMBUCINI

UNIVERSITY OF PERUGIA

DEPARTMENT OF MATHEMATICS AND COMPUTER SCIENCES

1, Via VANVITElLi, 06123 Perugia, ItAly

ORCID: 0000-0003-0161-8729

E-mail address: anna.sambucini@unipg.it

GIANLUCA VINTI

UNIVERSITY OF PERUGIA

DepartMENT OF MATHEMATICS AND COMPUTER SCIENCES

1, Via VANVITElli, 06123 Perugia, ItAly

ORCID: 0000-0002-9875-2790

E-mail address: gianluca.vinti@unipg.it 